\title{
Dosage of isoniazid and rifampicin poorly predicts drug exposure in tuberculosis patients
}

\author{
To the Editor:
}

Treatment of tuberculosis (TB) has been increasingly challenged by the emergence of drug resistance, toxicity, relapse and nonresponse [1]. Pharmacokinetic variability is a major driver of emerging drug resistance [2]. Risk of treatment failure was almost nine-fold higher in patients with low drug exposure compared to patients with higher drug exposure [3].

The World Health Organization (WHO) advises that adult patient dose should be based on total body weight with a maximum of $300 \mathrm{mg}$ once daily for isoniazid and $600 \mathrm{mg}$ for rifampicin [4]. The guidelines do not indicate whether total body weight used should be at the time of start of treatment or before the patient developed TB with subsequent weight loss.

A low body mass index (BMI) was associated with delay in sputum conversion and increased early mortality $[5,6]$. TB and malnutrition are closely associated; malnutrition is a risk factor for developing TB, while TB in turn causes malnutrition [7].

Due to the variability in clinical condition related to low total body weight, we hypothesised that total body weight may not be the best parameter for the selection of isoniazid and rifampicin dose. Indeed, other size descriptors might be better suited for dosing. Furthermore, other factors, such as liver enzymes or infection-related parameters, may influence drug exposure. The aim of this study was to investigate the association of different patient-related parameters with drug exposure in TB patients.

Patients aged $\geqslant 18$ years were included in the study if they were treated with rifampicin or isoniazid for TB between 2010 and 2014 at the Tuberculosis Centre Beatrixoord (Haren, The Netherlands). After a minimum of 2 weeks of treatment, a pharmacokinetic curve, consisting of a pre-dose and between three and nine time points selected randomly at $0.5-8 \mathrm{~h}$ post-dose, was obtained for therapeutic drug monitoring (TDM) as part of routine patient care. The pre-dose level was obtained just before dosing and this level was also used as the concentration level at $24 \mathrm{~h}$. Plasma samples were analysed for isoniazid or rifampicin by validated liquid chromatography-tandem mass spectrometry methods. Drug exposure was calculated as area under the concentration-time curve over $24 \mathrm{~h}$ in steady state (AUC0-24h). Demographic medical and biochemistry data were collected from the medical charts of patients including age, sex, total body weight, height, diagnosis, localisation of TB, drug dose, ethnicity, comorbidities and comedication. Biochemistry data included liver enzymes, renal function, albumin level, C-reactive protein (CRP) and erythrocyte sedimentation rate. The size descriptors BMI, body surface area, ideal body weight and fat-free mass were calculated using the demographic data recorded in the medical charts [8]. This study was evaluated by the local ethics committee and the need for informed consent was waived due to its retrospective nature (decision 2013-492).

We assessed the correlation of $\mathrm{AUC} 0-24 \mathrm{~h}$ of isoniazid and rifampicin with factors that can influence the pharmacokinetics of the drugs, such as age, size descriptors or biochemistry data. Based on the outcome of univariate and multivariate analyses, we compared both drug doses/total body weight and drug exposure of isoniazid and rifampicin between different patient groups. Two-sided p-values $\leqslant 0.05$ were considered statistically significant. All statistical analyses were performed using SPSS for Windows (version 22; IBM Corp., Armonk, NY, USA).

66 patients were included, of whom 55 had a pharmacokinetic curve for TDM of isoniazid and 63 patients underwent TDM of rifampicin. Median BMI was 17.9 (interquartile range 15.4-21.9) $\mathrm{kg} \cdot \mathrm{m}^{-2}$, indicating that the majority of these TB patients suffered from moderate to severe underweight.

Univariate analysis showed no association between isoniazid dose/total body weight and exposure (adjusted $\mathrm{R}^{2}=0.008, \mathrm{p}=0.232$ ). A weak positive association was found between rifampicin dose/total body weight and exposure (adjusted $\mathrm{R}^{2}=0.102, \mathrm{p}=0.006$ ) and between sex and exposure (adjusted $\mathrm{R}^{2}=0.194, \mathrm{p}<0.001$ ).

The comparisons of dose/total body weight and corresponding exposures in patient groups with different BMI, sex, relapse, CRP, acetylator status (isoniazid only) and the presence of comedication are presented in table 1. Table 1 illustrates that the effect of BMI resulted in comparable exposure, although the low BMI group $\left(<16 \mathrm{~kg} \cdot \mathrm{m}^{-2}\right)$ received a significantly higher dose/total body weight of both rifampicin and isoniazid. 
For isoniazid, an 18-fold difference was observed between the highest and lowest AUC0-24h. For rifampicin, a striking 56-fold difference was noted (table 1). These very wide ranges were not anticipated. Although many factors, such as nonlinear pharmacokinetics, concomitant food intake, comorbidities and gastrointestinal disorders may influence drug exposure [9-11], this is not addressed in current dosing guidelines.

The most outstanding outcome of this retrospective study is that dose/total body weight showed no correlation with isoniazid exposure and a weak but significant correlation with rifampicin exposure.

Patients receiving isoniazid were divided into fast and slow acetylators, based on the calculated half-life of isoniazid [12]. Results on isoniazid exposure presented in table 1 show no significant difference between fast and slow acetylators, which contrasts with what is generally experienced [11]. Combined with the low maximum concentrations we observed in our population, this might indicate that the main problem of low exposure in the patients we studied might have been caused by poor intestinal absorption rather than an increased rate of elimination. Our group recently performed a pharmacokinetic trial in treatment-naive TB patients, in which we also observed low maximum concentrations, indeed suggesting that reduced absorption might be an important cause of low exposure [10].

To date, TDM is only used in referral centres, which makes translation to outpatients difficult. Furthermore, outcome data are lacking because patients were transferred to municipal healthcare providers throughout the country for continuation of their treatment once they had improved enough clinically to be discharged from the TB clinic. Moreover, if low drug exposure was observed, dosages were increased, making it difficult to relate low drug exposure to poor outcome.

At this time, we were unable to identify factors predisposing to low exposure of isoniazid or rifampicin in TB patients. Therefore, monitoring drug exposure in patients at risk for altered drug exposure should still be undertaken [9]. Comorbidities such as HIV and diabetes mellitus, gastrointestinal disorders and concomitant food intake all have their impact on the wide interindividual variability of isoniazid and rifampicin [9, 10]. Isoniazid is metabolised primarily by the enzyme $N$-acetyltransferase- 2 . Due to its genetic polymorphism, patients can be classified as slow, fast or intermediate acetylators [13]. This metabolism combined with isoniazid's early bactericidal activity that is restricted to the first 2-4 days of treatment are reasons to perform TDM in the first days of treatment [13]. However, complete auto-induction of rifampicin takes $\geqslant 20$ days, as hepatic clearance almost doubles from baseline to steady state with a half-life of 4.5 days [14]. TB clinicians should take all these factors into account and perform TDM in these patients earlier, as nonresponse may be too late. If the auto-inductive effect of rifampicin is corrected for, TDM can be performed even after the first dose of isoniazid and rifampicin.

A practical programmatic approach to guide TB clinicians was recently published [1]. Referral of patients to a TB centre, based on clinical condition, may differ per setting, country and WHO region. To make TDM more easily accessible, our group recently suggested a way of facilitating the availability of TDM in resource-constrained areas with a high TB burden [15].

TABLE 1 Isoniazid and rifampicin dosing and exposure in different patient groups

\begin{tabular}{|c|c|c|c|c|c|c|}
\hline \multirow[b]{2}{*}{ Isoniazid } & \multicolumn{2}{|c|}{ Dose/TBW $\mathrm{mg} \cdot \mathrm{kg}^{-1}$} & \multirow[t]{2}{*}{ p-value ${ }^{\#}$} & \multicolumn{2}{|c|}{$A U C_{0-24 h} \mathrm{mg} \cdot \mathrm{h} \cdot \mathrm{L}^{-1}$} & \multirow[t]{2}{*}{$p$-value $\#$} \\
\hline & Yes & No & & Yes & No & \\
\hline Low dose $/$ TBW $\leqslant 5 \mathrm{mg} \cdot \mathrm{kg}^{-1}$ & $4.3(3.4-5.0)$ & $5.9(5.0-7.2)$ & $<0.001$ & $9.9(2.3-41.1)$ & $13.7(4.9-42.1)$ & 0.189 \\
\hline Male & $5.0(3.4-7.2)$ & $5.4(3.5-7.1)$ & 0.263 & $11.0(3.4-42.1)$ & $12.9(2.3-41.1)$ & 0.317 \\
\hline $\mathrm{BMI}<16 \mathrm{~kg} \cdot \mathrm{m}^{-2}$ & $5.7(4.6-7.1)$ & $4.9(3.4-7.2)$ & 0.003 & $12.1(3.4-42.1)$ & $11.6(2.3-41.1)$ & 0.868 \\
\hline Elevated $\mathrm{CRP} \geqslant 5 \mathrm{mg} \cdot \mathrm{L}^{-1}$ & $5.1(3.5-7.2)$ & $5.2(3.4-7.1)$ & 0.767 & $12.7(4.2-42.1)$ & $7.3(2.3-13.1)$ & 0.015 \\
\hline Rifampicin & Yes & No & & Yes & No & \\
\hline Low dose $/$ TBW $\leqslant 10 \mathrm{mg} \cdot \mathrm{kg}^{-1}$ & $8.9(6.9-10.0)$ & $11.7(10.1-20.5)$ & $<0.001$ & $29.4(2.3-114)$ & $39.8(12.8-130)$ & 0.026 \\
\hline Male & $10.0(6.9-20.5)$ & $10.7(7.1-14.4)$ & 0.044 & $28.0(2.3-67.9)$ & $46.9(18.7-130)$ & 0.002 \\
\hline $\mathrm{BMI}<16 \mathrm{~kg} \cdot \mathrm{m}^{-2}$ & $11.9(9.6-20.5)$ & $9.6(6.9-14.4)$ & $<0.001$ & $36.7(12.8-81.7)$ & $33.6(2.3-130)$ & 0.521 \\
\hline Elevated $\mathrm{CRP} \geqslant 5 \mathrm{mg} \cdot \mathrm{L}^{-1}$ & $10.3(7.1-20.5)$ & $9.8(6.9-12.8)$ & 0.579 & $33.5(2.3-130)$ & $34.0(12.8-64.3)$ & 0.655 \\
\hline Relapse & $9.7(7.4-12.3)$ & $10.4(6.9-20.5)$ & 0.661 & $39.2(13.7-81.7)$ & $34.1(2.3-130)$ & 0.513 \\
\hline
\end{tabular}

Data are presented as geometric mean (range), unless otherwise stated. TBW: total body weight; AUC0-24h: area under the concentration-time curve over $24 \mathrm{~h}$ in steady state; BMI: body mass index; CRP: C-reactive protein. ${ }^{\text {\# }}$ : data from comparisons of groups were tested using the MannWhitney U-test. 
In conclusion, dose/total body weight showed no correlation with isoniazid exposure and a weak but significant correlation with rifampicin exposure. Patients with a BMI $<16 \mathrm{~kg} \cdot \mathrm{m}^{-2}$ received a higher dose/ total body weight, but showed similar exposure to patients with a higher BMI. As patient characteristics predict drug exposure poorly, we advocate TDM in patients with HIV, diabetes mellitus, gastrointestinal disorders and those not responding to treatment, to optimise drug exposure and thereby lower the risk of treatment failure. in certain patients http://ow.ly/37523023n23

Marieke G.G. Sturkenboom ${ }^{1}$, Onno W. Akkerman ${ }^{2,3}$, Richard van Altena ${ }^{2}$, Wiel C.M. de Lange ${ }^{2,3}$, Jos G.W. Kosterink ${ }^{1,4}$, Tjip S. van der Werf ${ }^{3,5}$ and Jan-Willem C. Alffenaar ${ }^{1}$

${ }^{1}$ University of Groningen, University Medical Center Groningen, Dept of Clinical Pharmacy and Pharmacology, Groningen, The Netherlands. ${ }^{2}$ University of Groningen, University Medical Center Groningen, Tuberculosis Centre Beatrixoord, Haren, The Netherlands. ${ }^{3}$ University of Groningen, University Medical Center Groningen, Dept of Pulmonary Diseases and Tuberculosis, Groningen, The Netherlands. ${ }^{4}$ University of Groningen, Dept of Pharmacy, Pharmacotherapy and Pharmaceutical Care Section, Groningen, The Netherlands. ${ }^{5}$ University of Groningen, University Medical Center Groningen, Dept of Internal Medicine, Groningen, The Netherlands.

Correspondence: Jan-Willem C. Alffenaar, Dept of Clinical Pharmacy and Pharmacology, University Medical Center Groningen, University of Groningen, PO Box 30.001, 9700 RB Groningen, The Netherlands.

E-mail: j.w.c.alffenaar@umcg.nl

Received: Feb 022016 | Accepted after revision: June 302016 | First published online: Aug 042016

Conflict of interest: None declared.

\section{References}

1 van der Burgt EP, Sturkenboom MG, Bolhuis MS, et al. End TB with precision treatment! Eur Respir J 2016; 47: 680-682.

2 Srivastava S, Pasipanodya JG, Meek C, et al. Multidrug-resistant tuberculosis not due to noncompliance but to between-patient pharmacokinetic variability. J Infect Dis 2011; 204: 1951-1959.

3 Pasipanodya JG, McIlleron H, Burger A, et al. Serum drug concentrations predictive of pulmonary tuberculosis outcomes. J Infect Dis 2013; 208: 1464-1473.

4 World Health Organization. Guidelines for Treatment of Tuberculosis. 4th Edn. Geneva, World Health Organization, 2010. www.who.int/tb/publications/2010/9789241547833/en/

5 Zachariah R, Spielmann MP, Harries AD, et al. Moderate to severe malnutrition in patients with tuberculosis is a risk factor associated with early death. Trans R Soc Trop Med Hyg 2002; 96: 291-294.

6 Putri FA, Burhan E, Nawas A, et al. Body mass index predictive of sputum culture conversion among MDR-TB patients in Indonesia. Int J Tuberc Lung Dis 2014; 18: 564-570.

$7 \quad$ Gupta KB, Gupta R, Atreja A, et al. Tuberculosis and nutrition. Lung India 2009; 26: 9-16.

8 Green B, Duffull SB. What is the best size descriptor to use for pharmacokinetic studies in the obese? $\mathrm{Br} \mathrm{J}$ Clin Pharmacol 2004; 58: 119-133.

9 Alsultan A, Peloquin CA. Therapeutic drug monitoring in the treatment of tuberculosis: an update. Drugs 2014; 74: 839-854.

10 Saktiawati AM, Sturkenboom MG, Stienstra Y, et al. Impact of food on the pharmacokinetics of first-line anti-TB drugs in treatment-naive TB patients: a randomized cross-over trial. J Antimicrob Chemother 2016; 71: 703-710.

11 Pasipanodya JG, Srivastava S, Gumbo T. Meta-analysis of clinical studies supports the pharmacokinetic variability hypothesis for acquired drug resistance and failure of antituberculosis therapy. Clin Infect Dis 2012; 55: 169-177.

12 Tostmann A, Mtabho CM, Semvua HH, et al. Pharmacokinetics of first-line tuberculosis drugs in Tanzanian patients. Antimicrob Agents Chemother 2013; 57: 3208-3213.

13 Egelund EF, Alsultan A, Peloquin CA. Optimizing the clinical pharmacology of tuberculosis medications. Clin Pharmacol Ther 2015; 98: 387-393.

14 Chirehwa MT, Rustomjee R, Mthiyane T, et al. Model-based evaluation of higher doses of rifampin using a semimechanistic model incorporating autoinduction and saturation of hepatic extraction. Antimicrob Agents Chemother 2016; 60: 487-494.

15 Ghimire S, Bolhuis MS, Sturkenboom MGG, et al. Incorporating therapeutic drug monitoring into the World Health Organization hierarchy of tuberculosis diagnostics. Eur Respir J 2016; 47: 1867-1869. 